\title{
IncRNA BANCR promotes EMT in PTC via the Raf/MEK/ERK signaling pathway
}

\author{
YUANYUAN WANG ${ }^{1 *}$, JIAOJIAO GU $^{1 *}$, XIANGDE LIN $^{2}$, WEI YAN ${ }^{1}$, WENCHAO YANG $^{2}$ and GUOYANG WU ${ }^{1}$ \\ ${ }^{1}$ Department of General Surgery, Zhongshan Hospital, Xiamen University, Xiamen, Fujian 361000; \\ ${ }^{2}$ Digestive Diseases Center of Xiamen University Affiliated Zhongshan Hospital, Xiamen, Fujian 361004, P.R. China
}

Received May 26, 2017; Accepted December 20, 2017

DOI: $10.3892 / \mathrm{ol} .2018 .8017$

\begin{abstract}
Thyroid cancer is one of the most common types of cancer in the endocrine system. Among all types of thyroid cancer, papillary thyroid carcinoma (PTC) is the most common type of thyroid cancer. Long non-coding RNA (lncRNA) BRAF-activated non-protein-coding RNA (BANCR) is a 688-bp-long nucleotide transcript, which was first identified in melanoma. The function of BANCR in thyroid cancer remains unclear. The aim of the present study was to investigate whether BANCR is involved in the development of thyroid cancer. The results indicated that BANCR expression was increased in thyroid tumors compared with in adjacent normal tissues. Among cancer cell lines, the expression level of BANCR differed: BANCR expression in BCPAP cell lines was lower compared with that in CAL-62, WRO and FTC-133 cell lines. Overexpression of BANCR promoted the migration and invasion of BCPAP cells. Additionally, BANCR mediated epithelial-mesenchymal transition (EMT) by regulating the expression of epithelial (E)-cadherin, vimentin and neuronal $(\mathrm{N})$-cadherin. Overexpression of BANCR in BCPAP cells decreased the expression of E-cadherin and increased the expression of vimentin, $\mathrm{N}$-cadherin, phospho (p)-c-Raf, p-extracellular-signal-regulated kinase (ERK)/mitogen activated protein kinase (MEK)1/2 and p-ERK1/2. Administration of U0126 inhibitor inhibited the regulation of phosphorylation levels by MEK1/2 and ERK1/2. Additionally, U0126 upregulated the expression of E-cadherin and downregulated the expression of vimentin. Taken together, the results of the
\end{abstract}

Correspondence to: Dr Guoyang Wu, Department of General Surgery, Zhongshan Hospital, Xiamen University, 201 Hubin Road, Xiamen, Fujian 361000, P.R. China

E-mail: wuguoyangmail@aliyun.com

*Contributed equally

Key words: papillary thyroid carcinoma, BRAF-activated non-protein coding RNA, epithelial-mesenchymal transition, Raf, mitogen-activated protein kinase kinase, extracellular-signal-regulated protein kinase present study suggest that BANCR induces EMT in PTC through the Raf/MEK/ERK signaling pathway.

\section{Introduction}

Thyroid cancer is one of the most common types of cancer in the endocrine system, with an increasing incidence since 1980 (1). Papillary thyroid carcinoma (PTC) accounts for $80 \%$ of all types of thyroid cancer (2). However, the molecular mechanisms underlying PTC remain unclear and biomarkers for PTC are required.

Long non-coding RNAs (lncRNAs), which are 200 nucleotides long, do not encode any proteins but instead function to regulate the expression of associated genes (3). lncRNAs are now recognized as regulators of tumorigenesis and tumor progression $(4,5)$. BRAF mutations are the most common type of mutation in the lesions of patients with PTC, occurring in $45 \%$ of cases (6). BRAF-activated non-protein coding RNA (BANCR) is a 693-bp-long transcript on chromosome 9. It is frequently overexpressed and may be involved in the migration of melanoma cells (7). In non-small cell lung cancer, BANCR promotes the migration and invasion of cancer cells (8) through the mitogen-activated protein kinase (MAPK) signaling pathway. Epithelial-mesenchymal transition (EMT) refers to the process of transformation of epithelial cells to a mesenchymal cell phenotype, and serves an important function in tumor invasion and metastasis, since it may promote cancer cell migration and invasion $(9,10)$. The association between BANCR and cellular migration, invasion, EMT and MAPK signaling in PTC remains unclear.

Since BANCR is involved in the proliferation of PTC cells (11), the aim of the present study was to investigate the molecular mechanisms underlying BANCR and EMT in PTC.

\section{Materials and methods}

Patients and tissue samples. A total of 27 patients who received surgical resection for PTC were reviewed from January 2015 to December 2016 at the Department of General Surgery, Zhongshan Hospital of Xiamen University (Xiamen, China). Following surgery, all tumors and paired tissues were frozen in liquid nitrogen and stored at $-80^{\circ} \mathrm{C}$ for future experiments. The study was approved by the Ethics Committee on Human 
Research of the Zhongshan Hospital of Xiamen University and all patients provided written informed consent.

Cell lines and cell culture. The human PTC cell line BCPAP was purchased from the Chinese Academy of Sciences (Beijing, China). The human undifferentiated thyroid carcinoma cell line CAL-62 was purchased from the Chinese Academy of Sciences. The human cell follicular thyroid carcinoma lines WRO and FTC-133 were purchased from Shanghai Honsun Biological Technology Co., Ltd. (Shanghai, China). Cells were cultured in RPMI-1640 medium, $100 \mathrm{U} / \mathrm{ml}$ penicillin and $100 \mathrm{mg} / \mathrm{ml}$ streptomycin (all from Gibco; Thermo Fisher Scientific, Inc., Waltham, MA, USA) at $37^{\circ} \mathrm{C}$ in a humidified atmosphere containing $5 \% \mathrm{CO}_{2}$.

Total RNA extraction and reverse transcription-polymerase chain reaction $(R T-q P C R)$. Total RNA from tissues and cells was extracted using RNAiso Plus (Takara Biotechnology Co., Ltd., Dalian, China). RNA was reverse-transcribed using the PrimeScript RT kit (Takara), according to the manufacturer's instructions. The cDNA was amplified using the SYBR Premix Ex TaqII (Takara Biotechnology Co., Ltd.). Relative expression values were calculated using the $2^{-\Delta \Delta \mathrm{Cq}}$ method $(12,13)$. Primers were obtained from Sangon Biotech (Shanghai, China) and the sequences are presented in Table I. The software used for analysis was LightCycler ${ }^{\circledR} 96$ (version 1.1.0.1320; Roche Diagnostics GmbH, Mannheim, Germany). The thermocycling conditions were as follows: Initial denaturation at $95^{\circ} \mathrm{C}$ for $10 \mathrm{~min}$, followed by 45 cycles of denaturation at $95^{\circ} \mathrm{C}$ for $10 \mathrm{sec}$, annealing at $60^{\circ} \mathrm{C}$ for $30 \mathrm{sec}$, and a final cycle of denaturation at $95^{\circ} \mathrm{C}$ for $10 \mathrm{sec}$, annealing at $65^{\circ} \mathrm{C}$ for $60 \mathrm{sec}$ and extension at $97^{\circ} \mathrm{C}$ for $1 \mathrm{sec}$.

Establishment of stable cell lines. The BCPAP cell line was infected by lentivirus containing BCPAP-NC and BCPAP-BANCR constructs $\left[2 \times 10^{8}\right.$ transduction units (TU)/50 $\mu$; Genomeditech, Inc., Shanghai, China]. Then $1 \times 10^{6}$ BCPAP cells were transfected using lentivirus (final concentration $4 \times 10^{6} \mathrm{TU} / \mathrm{ml}$ ) and $6 \mu \mathrm{g} / \mathrm{ml}$ polybrene (Santa Cruz Biotechnology, Inc., Dallas, TX, USA) for $24 \mathrm{~h}$. The efficiency of infection was evaluated using RT-qPCR. Total RNA was extracted from BCPCP, BCPAP-NC and BCPAP-BANCR cell lines. The reference gene was $\beta$-actin and relevant primer sequences are presented in Table I. The instrument for RT-qPCR and thermocycling conditions were aforementioned. The method of quantification was $2^{-\Delta \Delta \mathrm{Cq}}$ (13).

Cell migration and invasion assays. For the migration experiments, BCPAP $\left(1.5 \times 10^{4}\right)$ cells were suspended in $200 \mu \mathrm{l}$ serum-free medium (RPMI-1640 medium; Gibco; Thermo Fisher Scientific, Inc.) and seeded into the upper chamber, whereas $800 \mu \mathrm{l}$ medium containing $10 \%$ fetal bovine serum was added to the lower chamber $\left(6.5 \mathrm{~mm}\right.$ Transwell ${ }^{\circledR}$ with $8.0 \mu \mathrm{m}$ Pore Polycarbonate Membrane Insert, Sterile; cat. no. 3422; Corning Incorporated, Corning, NY, USA). These cells were incubated for $14 \mathrm{~h}$, washed with PBS and fixed for between 15 and $20 \mathrm{~min}$. Cells were washed with PBS and stained with $0.1 \%$ crystal violet at room temperature for $10 \mathrm{~min}$. The images were captured under a light microscope (magnification, x100; AxioVert.A1; Zeiss GmbH, Jena, Germany). Five random fields
Table I. Primer sequences.

\begin{tabular}{ll}
\hline Target & \multicolumn{1}{c}{ Primers } \\
\hline$\beta$-actin & F: 5'-ACTGGAACTGTGAAGGTGAC-3' \\
& R: 5'-GTGGACTTGGGCGAGGACTG-3' \\
Vimentin & F: 5'-GAGAACTTGGCCGTTGAAGC-3' \\
& R: 5'-GCTTCCTGTTGGTGGCAATC-3' \\
E-cadherin & F: 5'-TGCCCAGAAGATGAATAAGG-3 \\
& R: 5'-GTGTATGTGGCAATGCGTTC-3' \\
N-cadherin & F:5'-CTCCTATGAGTGCAACAGGAACG-3' \\
& R: 5'-TTGGATCAGTGTCATAATCAAGTG \\
& CTGTA-3' \\
BANCR & F: 5'-CCTTCTTGTAGGGTCTGGATTG-3' \\
& R: 5'-CATTGGTGCTGCAGTCTATTTC-3' \\
\hline
\end{tabular}

BANCR, BRAF-activated non-protein-coding RNA; E-cadherin, epithelial cadherin; N-cadherin, neuronal cadherin.

were captured and quantified using a double-blind method. For the invasion experiments, BCPAP $\left(4 \times 10^{4}\right)$ cells were cultured for $14 \mathrm{~h}$ and processed as aforementioned.

Western blot analysis. Cells were washed twice with ice-cold PBS and lyzed using radioimmunoprecipitation assay buffer (Beyotime Institute of Biotechnology, Shanghai, China) supplemented with a protease inhibitor (complete Mini EDTA-free tablets; Roche Applied Science, Pleasanton, CA, USA) and phenylmethylsulfonyl fluoride (Beyotime Institute of Biotechnology). Proteins were denatured at $100^{\circ} \mathrm{C}$ for $10 \mathrm{~min}$ and equal amounts of sample $(30 \mu \mathrm{g})$ were separated by SDS-PAGE (10\% gels). Electrophoresed proteins were then transferred onto polyvinylidene fluoride membranes (EMD Millipore, Billerica, MA, USA). The membranes were blocked with 5\% BSA Blocking Buffer (Huayueyang Biotechnology Co., Ltd., Beijing, China) at room temperature for $1 \mathrm{~h}$. The densitometric analysis for the quantification of the bands was performed using enhanced chemiluminescence (ECL) chromogenic substrate (WesternBright ECL; Advansta, Menlo Park, CA, USA) and ChemiDoc ${ }^{\mathrm{TM}}$ XRS System (Bio-Rad Laboratories, Inc., Hercules, CA, USA). $\beta$-actin was used as an endogenous control. Membranes were incubated with following primary antibodies at $4^{\circ} \mathrm{C}$ overnight: Epithelial (E)-cadherin (1:1,000; cat. no. 3195S), neuronal (N)-cadherin (1:1,000; cat. no. $13116 S)$, vimentin (1:1,000; cat. no. 5741S), c-Raf (1:2,000; cat. no. 53745S), mitogen-activated protein kinase (MAPK)/extracellular-signal-regulated protein kinase (ERK) kinase 1/2 (MEK1/2) (1:2,000; cat. no. 4694S), ERK1/2 (1:2,000; cat. no. 4370S), phospho (p)-c-Raf (1:2,000; cat. no. $9421 S)$, p-MEK1/2 (1:2,000; cat. no. 2338S), p-ERK1/2 (1:2,000; cat. no. 4370S), GAPDH $(1: 2,000$; cat. no. $5174 S)$ and $\beta$-actin (1:1,000; cat. no. 3700S) (all from Cell Signaling Technology, Inc., Danvers, MA, USA). The following secondary antibodies used were used: Horseradish peroxidase (HRP)-conjugated goat anti-mouse and HRP-conjugated goat anti-rabbit immunoglobulin G antibody (Jackson ImmunoResearch Laboratories, Inc., West Grove, PA, USA). 

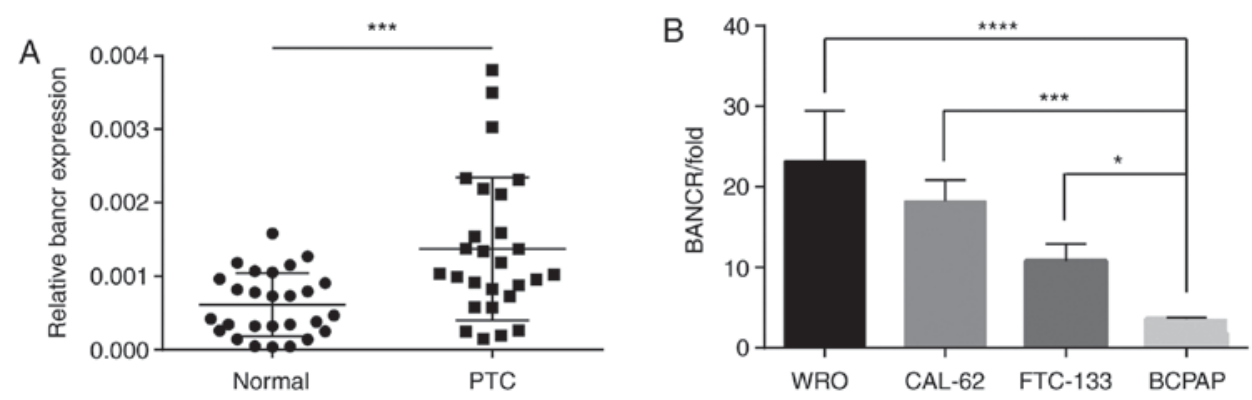

Figure 1. Expression levels of BANCR are significantly increased in PTC tissues compared with the control. (A) Expression level of BANCR in thyroid carcinoma and the adjacent normal tissue as assessed using RT-qPCR. The BANCR transcripts of tumors (PTC) and corresponding adjacent non-tumor tissues (normal) were analyzed using RT-qPCR and normalized to the internal control ( $\beta$-actin). (B) Relative BANCR levels in four thyroid cancer cell lines, including WRO, CAL-62, BCPAP and FTC-133. BCPAP cells exhibited the lowest expression levels of BANCR. $\mathrm{n}=3 .{ }^{*} \mathrm{P}<0.05,{ }^{* * * *} \mathrm{P}<0.001,{ }^{* * * *} \mathrm{P}<0.0001$. PTC, papillary thyroid carcinoma; BANCR, BRAF-activated non-protein-coding RNA; RT-qPCR, reverse transcription-quantitative polymerase chain reaction.

The inhibitor, U0126 (S1102; Selleck Chemicals, Houston, TX, USA), blocked the Raf-MEK-Erk signaling pathway by treating cells at $37^{\circ} \mathrm{C}$ for $30 \mathrm{~min}$. U0126 was diluted by DMSO (20688; Thermo Fisher Scientific, Inc.) to a final concentration of $20 \mu \mathrm{mol} / \mathrm{l}$

Confocal imaging. BCPAP cells were plated at $3 \times 10^{4}$ cells $/$ well. Cells were washed with PBS twice and fixed with methanol for $20 \mathrm{~min}$ at room temperature. Cells were washed again with PBS three times and cells were incubated at $37^{\circ} \mathrm{C}$ in a sealed box for $1 \mathrm{~h}$ with $5 \%$ bovine serum albumin (BSA) in PBS. Cells were then incubated with primary antibodies E-cadherin (1:150; cat. no. 3195S) and vimentin (1:200; cat. no. 5741S) (both from Cell Signaling Technology, Inc.) which were diluted with 5\% BSA to $80 \mu \mathrm{l}$ at $4^{\circ} \mathrm{C}$ overnight. Cells were then washed with PBS four times and then incubated with fluorescent-tag-labeled secondary antibodies (Alexa Fluor 555-phalloidin; 1:600; cat. no. 8953S; Cell Signaling Technology, Inc.) at $37^{\circ} \mathrm{C}$ for $1 \mathrm{~h}$ in the dark. Cells were washed with PBS four times and incubated with $100 \mu 1$ DAPI at room temperature for $10 \mathrm{~min}$. Cells were washed with PBS four times and then incubated with $8 \mu 1$ Mounting medium, antifading (Beijing Solarbio Science and Technology Co., Ltd., Beijing, China) and dried. Images of cells were captured using a Zeiss LSM 510 system (magnification, x100; Carl Zeiss AG, Oberkochen, Germany).

Statistical analysis. Data were analyzed using GraphPad Prism (version 5.0; GraphPad Software, Inc., La Jolla, CA, USA). The relevant data are expressed as the mean \pm standard error of the mean. Statistical significance among groups was assessed using Student's t-test, one-way analysis of variance followed by least significant difference method. $\mathrm{P}<0.05$ was considered to indicate a statistically significant difference.

\section{Results}

IncRNA BANCR expression levels in PTC tissues and cell lines. The expression level of BANCR in 27 paired tissue samples from patients with PTC was evaluated. The BANCR level was significantly increased in the thyroid tissues compared with the adjacent normal tissues (Fig. 1A). The expression of BANCR in four human thyroid cancer cell lines were also evaluated. The cell lines differed in the expression levels of BANCR (Fig. 1B). The BCPAP cell line had the lowest expression level of BANCR and was therefore employed for subsequent experiments.

IncRNA BANCR promotes migration and invasion of PTC cells. BCPAP cells were transfected with lentivirus and the expression levels of BANCR were assessed. BANCR was significantly upregulated in BCPAP-BANCR cells compared with the BCPAP or BCPAP-NC (Fig. 2A). The migration and invasion of BCPAP-BANCR cells were significantly increased compared with the cells transfected with empty vector (Fig. 2B).

IncRNA BANCR promotes EMT in PTC cell lines. The expression of EMT-induced markers, including E-cadherin, $\mathrm{N}$-cadherin and vimentin, was assessed in BCPAP cells using RT-qPCR, western blot analysis and confocal microscopy. The results indicated that upregulated expression of BANCR increased the expression levels of $\mathrm{N}$-cadherin and vimentin, but decreased the expression of E-cadherin (Fig. 3A). Western blot analysis and confocal microscopy also revealed that increased BANCR expression upregulated the expression of vimentin and decreased the expression of E-cadherin in BCPAP-BANCR cells (Fig. 3B and C).

IncRNA BANCR regulates EMT in PTC via the Raf/MEK/ERK signaling pathway. First, the expression levels of c-Raf, MEK1/2 and ERK1/2 were evaluated in the BCPAP-BANCR and BCPAP-NC cells using western blot analysis. The expression levels of $\mathrm{p}$-c-Raf, $\mathrm{p}$-MEK1/2 and p-ERK1/2 were significantly increased in BCPAP-BANCR cells compared with in BCPAP-NC cells (Fig. 4A). These results suggest an association between BANCR and the Raf/MEK/ERK signaling pathway. To test this hypothesis, BCPAP cells were treated with the c-Raf inhibitor U0126 and used the cells treated with DMSO as the blank control group. The results indicated that treatment with the c-Raf inhibitor U0126 inactivated p-MEK1/2 and p-ERK1/2 in BCPAP cell lines (Fig. 4B). Therefore, U0126 may inhibit the effect of BANCR on the Raf/MEK/ERK signaling pathway. In order to clarify the effects of BANCR in inducing EMT via the Raf/MEK/ERK 

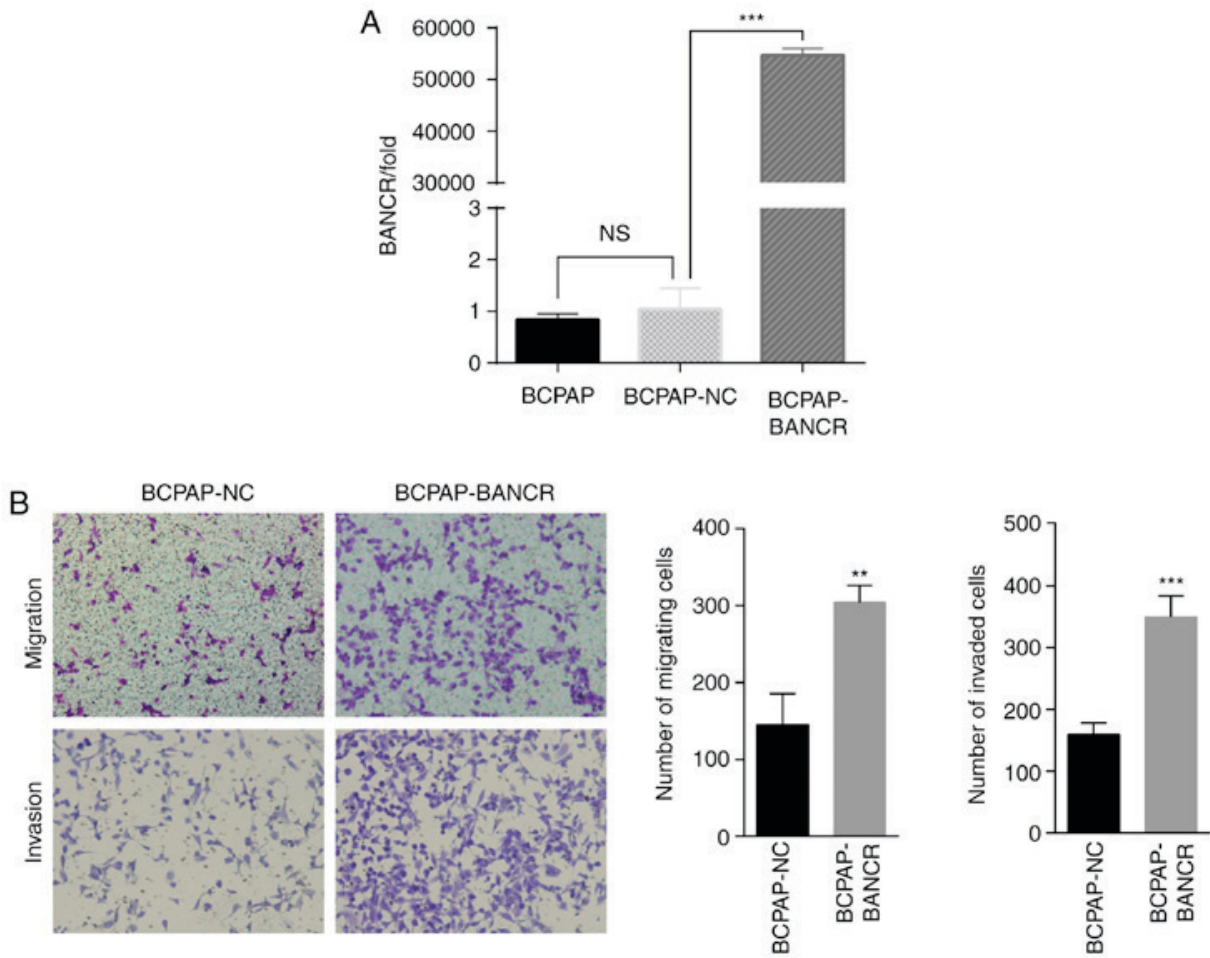

Figure 2. BANCR promotes cellular migration and invasion in PTC. (A) BANCR is overexpressed in the BCPAP-BANCR cell line following transfection of BCPAP cells with pcDNA-BANCR compared with the protocell BCPAP and the BCPAP-NC infected with a lentivirus vector without BANCR (B) BCPAP-BANCR cells exhibited significantly increased migration and invasion compared with BCPAP-NC cells ( $\mathrm{n}=3$; magnification, $\mathrm{x} 100)$. ${ }^{* *} \mathrm{P}<0.01$, ${ }^{* * *} \mathrm{P}<0.001$ vs. BCPAP-NC cells. PTC, papillary thyroid carcinoma; BANCR, BRAF-activated non-protein-coding RNA; NC, negative control; NS, not significant.
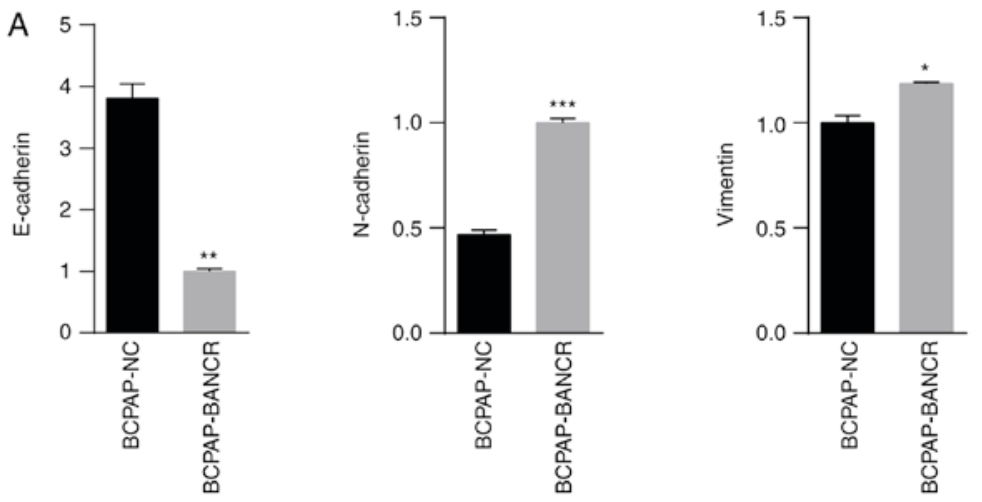

B
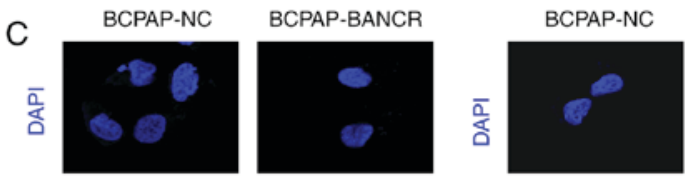

BCPAP-BANCR
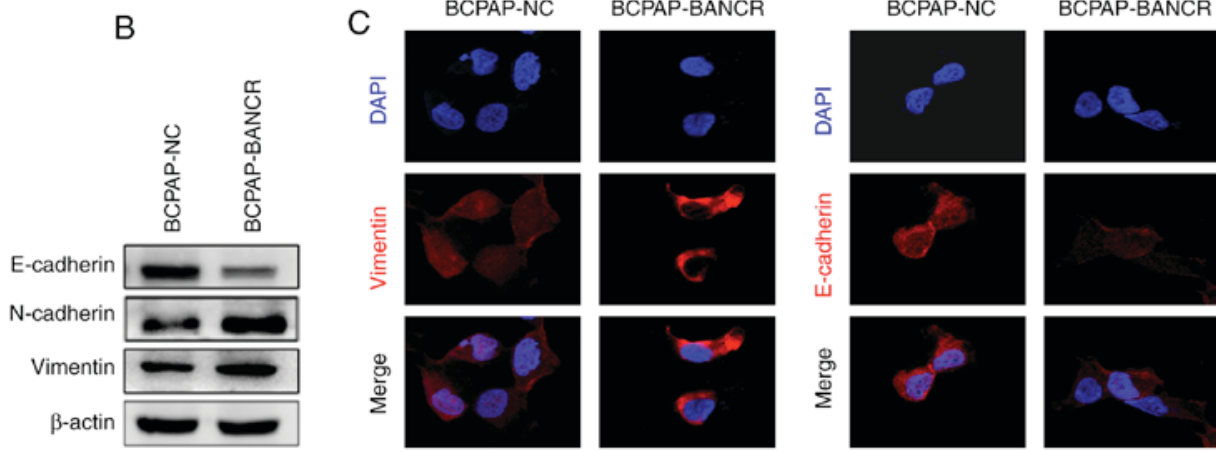

Figure 3. BANCR overexpression promotes cellular invasion and metastasis by inducing EMT in PTC. (A) The expression of N-cadherin and vimentin was upregulated, and the expression of E-cadherin was downregulated in BCPAP-BANCR cells compared with in BCPAP-NC cells. (B) Western blot analysis of the expression of E-cadherin, vimentin and N-cadherin in BCPAP-NC and BCPAP-BANCR cells. (C) Expression of E-cadherin, vimentin and N-cadherin in BCPAP-NC and BCPAP-BANCR cells as assessed using confocal microscopy. DAPI was used to stain the nucleus. Increased BANCR expression upregulated vimentin and downregulated E-cadherin in BCPAP-BANCR cells. ( $\mathrm{n}=3$; magnification, $\mathrm{x} 100)$. ${ }^{*} \mathrm{P}<0.05,{ }^{* *} \mathrm{P}<0.01,{ }^{* * *} \mathrm{P}<0.001$ vs. BCPAP-NC cells. PTC, papillary thyroid carcinoma; BANCR, BRAF-activated non-protein-coding RNA; NC, negative control; E-cadherin, epithelial cadherin; N-cadherin, neuronal cadherin. 


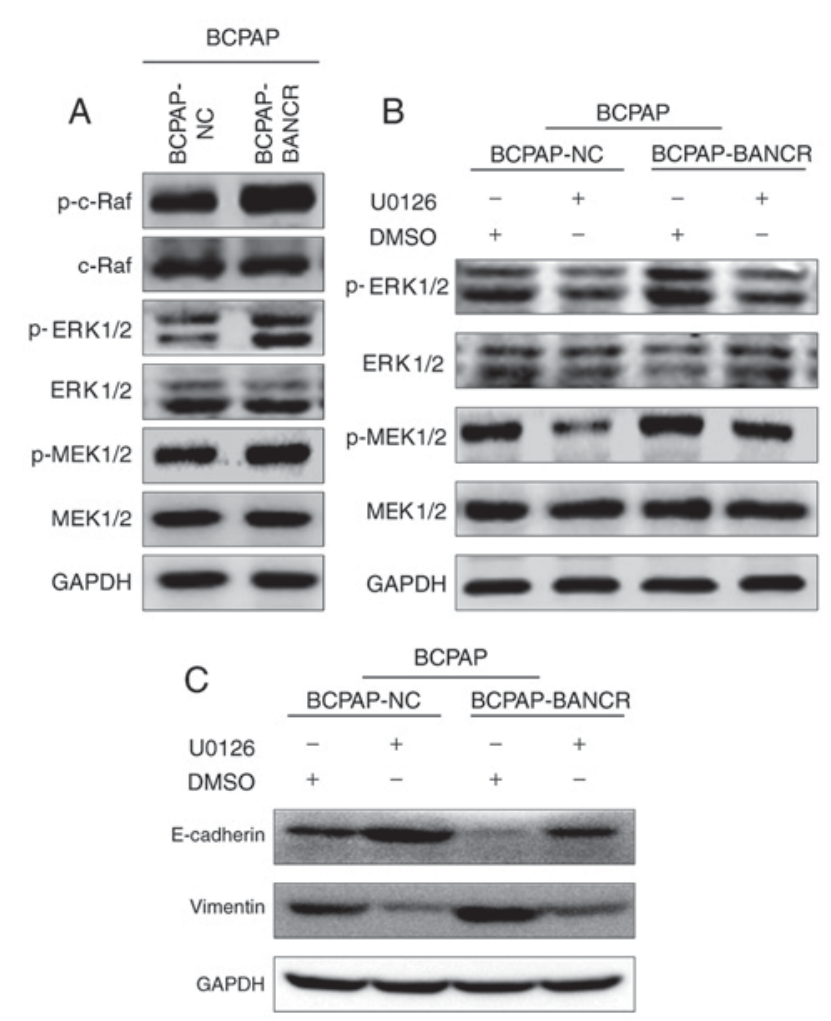

Figure 4. BANCR induces EMT in PTC via the Raf/MEK/ERK signaling pathway. (A) Overexpression of BANCR upregulated the expression of p-c-Raf, p-MEK1/2 and p-ERK1/2. (B) Following incubation with the c-Raf inhibitor U0126, the ability of BCPAP-BANCR cells to upregulate p-c-Raf, p-MEK1/2, p-ERK1/2 was diminished. (C) Following overexpression of BANCR, E-cadherin was downregulated in BCPAP cells. However, in response to U0126 treatment, E-cadherin expression was upregulated and vimentin expression was downregulated in BCPAP-BANCR cells compared with BCPAP-NC cells. PTC, papillary thyroid carcinoma; BANCR, BRAF-activated non-protein-coding RNA; NC, negative control; E-cadherin, epithelial cadherin; EMT, epithelial-mesenchymal transition; p-, phospho-; ERK, extracellular-signal-regulated protein kinase; MEK, mitogen-activated protein kinase/ERK kinase; DMSO, dimethyl sulfoxide.

signaling pathway, the BCPAP-NC and BCPAP-BANCR cell lines were treated with U0126 and the expression levels of E-cadherin and vimentin were assessed using western blot analysis (Fig. 4C). The results demonstrated that the expression of E-cadherin was downregulated following overexpression of BANCR in the BCPAP cell line. However, in response to U0126 treatment, E-cadherin expression was upregulated in both BCPAP-NC and BCPAP-BANCR cell lines, vimentin expression was also upregulated following the overexpression of BANCR; however, the expression was downregulated when treated with U0126 in both BCPAP and BCPAP-BANCR cell lines (Fig. 4C). Therefore, BANCR may induce EMT in PTC via the Raf/MEK/ERK signaling pathway.

\section{Discussion}

lncRNAs were traditionally considered to exhibit no cellular function since they do not encode any proteins. Recent studies have confirmed their function in biological processes, including regulating or controlling gene expression, and in pathological processes, including tumorigenesis $(14,15)$. It has been identified that IncRNAs affect numerous cellular processes in tumor cells, including the cell cycle, survival rate, proliferation and migration (16-19).

In thyroid cancer, a number of IncRNAs demonstrate differential expression between carcinoma and para-cancer tissues (20). For example, maternal expressed gene 3 (MEG3) was the first lncRNA demonstrated to act as a tumor suppressor in melanoma cells (21). In PTC, MEG3 was upregulated in carcinoma tissues compared with normal tissues and suppressed migration and invasion by targeting Ras-related C3 botulinum toxin substrate 1 (22). Papillary thyroid carcinoma susceptibility candidate 3 acts as a tumor suppressor in thyroid cancer cells and leads to marked significant inhibition of proliferation, cell cycle arrest and increased apoptosis (23). Antisense non-coding RNA in the INK4 locus has been demonstrated to promote the invasion and metastasis of thyroid cancer cells through the transforming growth factor- $\beta /$ small mother against decapentaplegic signaling pathway (24). Nc886 exerts an oncogenic function in thyroid cancer by suppressing double-stranded RNA-activated protein kinase (25).

BANCR participates in the proliferation of malignant melanoma cells (7). BANCR regulates cellular proliferation and migration via p38 MAPK and c-Jun N-terminal kinase inactivation in lung carcinoma (LC) (8). In lung cancer cells, BANCR is associated with poor prognosis and promotes metastasis by inducing EMT (26). Recently, BANCR has been demonstrated to promote cell proliferation in PTC (11), However, the association between BANCR and EMT and the underlying molecular mechanism in PTC remain unclear. To the best of our knowledge, the present study is the first to address these key issues.

The results of the present study demonstrated that the expression of BANCR in 27 paired tissue samples from PTC patients exhibited a significant increase compared with its expression in the adjacent normal tissues. The BCPAP cell line was selected for subsequent experiments due to low expression of BANCR and it was demonstrated that upregulation of BANCR expression may promote the migration and invasion of PTC cells. Additionally, the expression of EMT-induced markers (E-cadherin, N-cadherin and vimentin) in cells overexpressing BANCR was also assessed using qPCR, western blot analysis and confocal microscopy. The results indicated that increased BANCR expression levels were associated with increased expression of $\mathrm{N}$-cadherin and vimentin. Therefore, it was hypothesized that BANCR may promote EMT in PTC. In thyroid cancer, there are two classical cell signaling pathways: The ERK/MAPK signaling pathway and the phosphoinositide 3-kinase/protein kinase B signaling pathway. A number of studies have demonstrated that the V600E mutation of BRAF activates the MAPK signaling pathway (27). BANCR has previously been reported to be associated with BRAF (V600E) and BANCR regulated LC proliferation and migration via the MAPK signaling pathway (8). The results of the present study indicated that overexpression of BANCR may upregulate the expression of p-c-Raf, p-MEK1/2 and p-ERK1/2. Therefore, BANCR may activate the Raf/MEK/ERK signaling pathway. This effect was reversed by U0126 treatment. In BCPAP-NC and BCPAP-BANCR cells, the expression of E-cadherin was upregulated, whereas vimentin expression was downregulated in response to U0126 treatment. 
The present study demonstrated that BANCR promotes the migration, invasion and EMT in PTC via the Raf/MEK/ERK signaling pathway. Although the BCPAP cell line was the only cell line employed, the results of the present study of value. In the future, further tissue samples from patients with PTC and additional cell lines may be utilized to confirm the results of the present study. Additionally, in vivo experiments employing nude mice may also provide new insights into the function of BANCR on PTC.

\section{Acknowledgements}

The present study was supported by the Department of General Surgery, Zhongshan Hospital. The authors thank the Digestive Diseases Center of Xiamen City for support.

\section{Competing interests}

The authors declare that they have no competing interests.

\section{References}

1. Chen AY, Jemal A and Ward EM: Increasing incidence of differentiated thyroid cancer in the United States, 1988-2005. Cancer 115: 3801-3807, 2009.

2. Wang Y, Guo Q, Zhao Y, Chen J, Wang S, Hu J and Sun Y: BRAF-activated long non-coding RNA contributes to cell proliferation and activates autophagy in papillary thyroid carcinoma. Oncol Lett 8: 1947-1952, 2014.

3. Ponting CP, Oliver PL and Reik W: Evolution and functions of long noncoding RNAs. Cell 136: 629-641, 2009.

4. Zhu L and Xu PC: Downregulated lncRNA-ANCR promotes osteoblast differentiation by targeting EZH2 and regulating Runx2 expression. Biochem Biophys Res Commun 432: 612-617, 2013.

5. Nakagawa T, Endo H, Yokoyama M, Abe J, Tamai K, Tanaka N, Sato I, Takahashi S, Kondo T and Satoh K: Large noncoding RNA HOTAIR enhances aggressive biological behavior and is associated with short disease-free survival in human non-smal cell lung cancer. Biochem Biophys Res Commun 436: 319-324, 2013.

6. Xing M: BRAF mutation in thyroid cancer. Endocr Relat Cancer 12: 245-262, 2005.

7. Li R, Zhang L, Jia L, Duan Y, Li Y, Bao L and Sha N: Long non-coding RNA BANCR promotes proliferation in malignant melanoma by regulating MAPK pathway activation. PLoS One 9: e100893, 2014.

8. Jiang W, Zhang D, Xu B, Wu Z, Liu S, Zhang L, Tian Y, Han X and Tian D: Long non-coding RNA BANCR promotes proliferation and migration of lung carcinoma via MAPK pathways. Biomed Pharmacother 69: 90-95, 2015.

9. Przybyla L, Muncie JM and Weaver VM: Mechanical control of epithelial-to-mesenchymal transitions in development and cancer. Annu Rev Cell Dev Biol 32: 527-554, 2016.

10. Thiery JP, Acloque H, Huang RY and Nieto MA: Epithelial-mesenchymal transitions in development and disease. Cell 139: 871-890, 2009.
11. Zheng $\mathrm{H}$, Wang M, Jiang L, Chu H, Hu J, Ning J, Li B, Wang D and Xu J: BRAF-activated long noncoding RNA modulates papillary thyroid carcinoma cell proliferation through regulating thyroid stimulating hormone receptor. Cancer Res Treat 48: 698-707, 2016.

12. Schmittgen TD and Livak KJ: Analyzing real-time PCR data by the comparative C(T) method. Nat Protoc 3: 1101-1108, 2008.

13. Livak KJ and Schmittgen TD: Analysis of relative gene expression data using real-time quantitative PCR and the 2(-Delta Delta C(T)) method. Methods 25: 402-408, 2001.

14. Flockhart RJ, Webster DE, Qu K, Mascarenhas N, Kovalski J, Kretz M and Khavari PA: BRAFV600E remodels the melanocyte transcriptome and induces BANCR to regulate melanoma cell migration. Cancer Res 22: 1006-1014, 2012

15. Gupta RA, Shah N, Wang KC, Kim J, Horlings HM, Wong DJ, Tsai MC, Hung T, Argani P, Rinn JL, et al: Long non-coding RNA HOTAIR reprograms chromatin state to promote cancer metastasis. Nature 464: 1071-1076, 2010.

16. Xia M, Yao L, Zhang Q, Wang F, Mei H, Guo X and Huang W: Long noncoding RNA HOTAIR promotes metastasis of renal cell carcinoma by up-regulating histone $\mathrm{H} 3 \mathrm{~K} 27$ demethylase JMJD3. Oncotarget 8: 19795-19802, 2017.

17. Yang F, Bi J, Xue X, Zheng L, Zhi K, Hua J and Fang G: Up-regulated long non-coding RNA H19 contributes to proliferation of gastric cancer cells. Febs J 279: 3159-3165, 2012.

18. Yang C, Li X, Wang Y, Zhao L and Chen W: Long non-coding RNA UCA1 regulated cell cycle distribution via CREB through P13-K dependent pathway in bladder carcinoma cells. Gene 496: 8-16, 2012.

19. Esteller M: Non-coding RNAs in human disease. Nat Rev Genet 12: 861-874, 2011.

20. Lan X, Zhang H, Wang Z, Dong W, Sun W, Shao L, Zhang T and Zhang D: Genome-wide analysis of long noncoding RNA expression profile in papillary thyroid carcinoma. Gene 569: 109-117, 2015.

21. Gibb EA, Brown CJ and Lam WL: The functional role of long non-coding RNA in human carcinomas. Mol Cancer 10: 38, 2011.

22. Wang C, Yan G, Zhang Y, Jia X and Bu P: Long non-coding RNA MEG3 suppresses migration and invasion of thyroid carcinoma by targeting of Rac1. Neoplasma 62: 541-549, 2015.

23. Fan M, Li X, Jiang W, Huang Y, Li J and Wang Z: A long non-coding RNA, PTCSC3, as a tumor suppressor and a target of miRNAs in thyroid cancer cells. Exp Ther Med 5: 1143-1146, 2013.

24. Zhao JJ, Hao S, Wang LL, Hu CY, Zhang S, Guo LJ, Zhang G, Gao B, Jiang Y, Tian WG and Luo DL: Long non-coding RNA ANRIL promotes the invasion and metastasis of thyroid cancer cells through TGF- $\beta /$ Smad signaling pathway. Oncotarget 7 : 57903-57918, 2016.

25. Lee EK, Hong SH, Shin S, Lee HS, Lee JS, Park EJ, Choi SS, Min JW, Park D, Hwang JA, et al: nc886, a non-coding RNA and suppressor of PKR, exerts an oncogenic function in thyroid cancer. Oncotarget 7: 75000-75012, 2016.

26. Sun M, Liu XH, Wang KM, Nie FQ, Kong R, Yang JS, Xia R, $\mathrm{Xu}$ TP, Jin FY, Liu ZJ, et al: Downregulation of BRAF activated non-coding RNA is associated with poor prognosis for non-small cell lung cancer and promotes metastasis by affecting epithelial-mesenchymal transition. Mol Cancer 13: 68, 2014.

27. Fraser S, Go C, Aniss A, Sidhu S, Delbridge L, Learoyd D, Clifton-Bligh R, Tacon L, Tsang V, Robinson B, et al: BRAF(V600E) mutation is associated with decreased disease-free survival in papillary thyroid cancer. World J Surg 40: 1618-1624, 2016. 